\title{
MicroRNA-133a-3p suppresses malignant behavior of non-small cell lung cancer cells by negatively regulating ERBB2
}

\author{
YANHUI XU, LEI ZHANG, LILONG XIA and XINHAI ZHU \\ Department of Thoracic Surgery, Zhejiang Hospital, Hangzhou, Zhejiang 310030, P.R. China
}

Received April 20, 2020; Accepted October 8, 2020

DOI: 10.3892/ol.2021.12718

\begin{abstract}
Non-small cell lung cancer (NSCLC) has high morbidity and mortality rates worldwide, and tumor metastasis is generally associated with poor prognosis. Chemotherapy resistance aggravates the challenges associated with treating NSCLC. Therefore, identifying effective targets and developing therapies based on these findings could bring novel perspectives for patients with metastatic NSCLC. The expression levels of receptor tyrosine-protein kinase erbB-2 (ERBB2) are associated with NSCLC progression. Differential microRNA (miR) expression profiles have been identified in tumors and can be used to identify multiple malignant phenotypes. miR-133a-3p expression is dysregulated in a variety of tumors. However, to the best of our knowledge, the association between miR-133a-3p and the NSCLC pathogenesis process has not been demonstrated yet. The present study revealed a decrease in miR-133a-3p expression in both tissues and cell lines, which was detected using reverse transcription-quantitative (RT-q)PCR, and western blotting and RT-qPCR demonstrated ERBB2 levels were increased at both protein and mRNA levels. Bioinformatics analysis and dual-luciferase reporter assays demonstrated that ERBB2 was a direct target of miR-133a-3p. Furthermore, MTT, wound healing and Transwell assays revealed that overexpression of miR-133a-3p suppressed proliferation, invasion and migration of NSCLC cells, respectively, effects that were inhibited following ERBB2 overexpression. In addition, immunofluorescence assays demonstrated that overexpression of ERBB2 upregulated $\mathrm{N}$-cadherin expression, while E-cadherin expression was downregulated. In conclusion, the present data demonstrated that miR-133a-3p acted as a tumor suppressor by negatively regulating ERBB2 expression. The miR-133a-3p/ERBB2 axis
\end{abstract}

Correspondence to: Dr Lilong Xia or Dr Xinhai Zhu, Department of Thoracic Surgery, Zhejiang Hospital, 1229 Gudun Road, Xihu, Hangzhou, Zhejiang 310030, P.R. China

E-mail: 1laxiu@yeah.net

E-mail: zhuxinhai0@163.com

Key words: non-small cell lung cancer, microRNA-133a-3p, erb-b2 receptor tyrosine kinase 2 , proliferation, invasion, migration may be a potential target for the diagnosis and treatment of NSCLC in the future.

\section{Introduction}

Lung cancer is a common malignant tumor with high morbidity and mortality rates $(1,2)$. In 2015, 158,040 patients succumbed to lung cancer in the United States (3). Non-small cell lung cancer (NSCLC) accounts for $87 \%$ of all lung cancers, and $40 \%$ of NSCLC cases are diagnosed with tumor metastasis (3). Although targeted therapies, such as gefitinib, are available for patients with gene mutations, including EGFR mutations and anaplastic lymphoma kinase rearrangements, these mutations are not common in NSCLC (4). Therefore, most patients are treated with conventional chemotherapy (5). Systemic chemotherapy can improve survival and alleviate disease-related symptoms. However, a large proportion of patients develop drug resistance after several chemotherapy courses, resulting in tumor relapse, invasion and metastasis $(6,7)$. Therefore, it would be of great clinical value to identify novel, potent targets for the treatment of NSCLC, and to explore their association with invasion and metastasis of NSCLC.

Receptor tyrosine-protein kinase erbB-2 (ERBB2) is a $185 \mathrm{kDa}$ cell membrane receptor encoded by the oncogene erbB-2, which is a member of the EGFR family. ERBB2 is involved in multiple cell events, such as proliferation, differentiation and apoptosis $(8,9)$. ERBB2 is highly expressed in multiple malignant tumors, such as breast cancer, ovarian cancer and hepatocellularcarcinoma (8,10-13). Downregulation of ERBB2 suppresses the proliferation and invasion of breast cancer and gastric cancer cells $(12,14,15)$. ERBB2 expression is positively associated with NSCLC, and high expression levels of ERBB2 are associated with resistance to radiotherapy and chemotherapy $(16,17)$. Therefore, ERBB2 may be a potential prognostic biomarker and therapeutic target for NSCLC.

MicroRNAs (miRNAs/miRs) are endogenous short non-coding sequences with a length of 20-24 bases. miRNAs serve a role in multiple biological processes, such as cell proliferation, differentiation and apoptosis, by regulating protein expression at the posttranscriptional level $(18,19)$. miRNAs regulate mRNAs by binding to the $3^{\prime}$ untranslated region (3'UTR) end of mRNA, degrading mRNA or repressing protein translation. miR-133a is highly conserved and widely expressed in various organisms, and can be expressed as the subtypes miR-133a-3p and miR-133a-5p (20). In a variety of 
cancer types, including gastric cancer, bladder cancer and oral squamous cell carcinoma, miR-133a-3p expression is downregulated (21-23). Yang et al (24) reported that patients with NSCLC with high miR-133a expression in tumor cells had a favorable prognosis. To the best of our knowledge, no reports have demonstrated the association between miR-133a-3p and NSCLC pathogenesis.

The present study aimed to investigate the association between dysregulated miR-133a-3p expression and NSCLC pathogenesis, and to explore the role of miR-133a-3p andERBB2 in NSCLC carcinogenesis. The expression of miR-133a-3p and ERBB2 in NSCLC tissues and cell lines was detected using reverse transcription-quantitative (RT-q)PCR and western blotting, respectively. The effects of miR-133a-3p on the malignant phenotypes of NSCLC cells were then evaluated by multiple experiments, such as MTT and Transwell assays, and the underlying mechanisms were further investigated. The present data indicate that the miR-133a-3p/ERBB2 axis could be a potential therapeutic target of NSCLC.

\section{Materials and methods}

Sample collection. Material was collected from 77 patients (45 males and 32 females, aged $66.58 \pm 14.38$ years) primarily diagnosed with NSCLC who underwent primary surgery at Zhejiang Hospital (Hangzhou, China) between January 2010 and January 2017. None of the participants received chemotherapy or radiotherapy before surgery and none of the participants was diagnosed other malignant diseases before. The present study was conducted in accordance with the Helsinki Declaration, and all patients or direct relatives consented to participate in the study. Furthermore, the present study was approved by the Ethics Committee of Zhejiang Hospital. After surgery, the tumor and adjacent healthy tissues ( $\geq 1-\mathrm{cm}$ from the edge of tumor) were collected and stored in liquid nitrogen $\left(-196^{\circ} \mathrm{C}\right)$.

Cell culture and transfection. The normal human lung epithelial cell line BEAS-2B and human NSCLC HCC827 and H1299 cell lines were purchased from the Institute of Shanghai Biochemistry and Cell Biology. BEAS-2B was cultured in BEBM medium (Gibco; Thermo Fisher Scientific, Inc.) supplemented with 10\% FBS (HyClone; Cyvita). HCC827 and H1299 cells were cultured in RPMI-1640 medium (Invitrogen; Thermo Fisher Scientific, Inc.) supplemented with 10\% FBS and $1 \%$ penicillin/streptomycin $(100 \mathrm{U} / \mathrm{ml}$ penicillin and $0.1 \mathrm{mg} / \mathrm{ml}$ streptomycin; Thermo Fisher Scientific, Inc.). All cells were cultured with $5 \% \mathrm{CO}_{2}$ at $37^{\circ} \mathrm{C}$.

For transfection, HCC827 and $\mathrm{H} 1299$ were collected and seeded in 6 -well plates at the density of $1 \times 10^{5} /$ well. After incubated for $24 \mathrm{~h}$, cells treated with $100 \mathrm{nM}$ mimic control (5'-CAGCUGGUUGAAGGGGACCAAA-3'; Shanghai GenePharma Co., Ltd.), 75 nM miR-133a-3p mimic (5'-UUU GGUCCCCUUCAACCAGCUG-3'; Shanghai GenePharma Co., Ltd.), $600 \mathrm{ng} /$ well pcDNAn empty vector negative control (pcDNA-NC; Shanghai GenePharma Co., Ltd.) or 600 ng/well pcDNA-ERBB2 (Shanghai GenePharma Co., Ltd.) using Lipofectamine 3000 (Invitrogen; Thermo Fisher Scientific, Inc.) according to the manufacturers' protocols. The $1 \mu \mathrm{g}$ nucleic acid fragment and $2 \mu$ l Lipofectamine 3000 were mixed with $1 \mathrm{ml}$ RPMI-1640 medium without serum, and then incubated for $15 \mathrm{~min}$ at room temperature. Then, the mixture was mixed with $4 \mathrm{ml}$ serum-free RPMI-1640 medium and added into plates. After cultured for $24 \mathrm{~h}$, the culture medium was removed and replaced with RPMI-1640 medium containing 10\% FBS. After 24-72 h, cells were used for subsequent experiments.

MTT assay. Following treatment with control mimics, miR-133a-3p mimics or pcDNA-ERBB2, H1299 and HCC827 cells were collected and seeded into 96-well plates at the density of $1 \times 10^{4}$ cells/well. After culturing for 24,48 and $72 \mathrm{~h}$, medium was removed and replaced with medium containing MTT (KGA312; Nanjing KeyGen Biotech Co., Ltd.) at a final concentration of $0.5 \mathrm{mg} / \mathrm{ml}$ and cells were cultured for another $4 \mathrm{~h}$ at $37^{\circ} \mathrm{C}$. Finally, the medium was replaced with $200 \mu \mathrm{l}$ DMSO. After $10 \mathrm{~min}$, the absorbance at $490 \mathrm{~nm}$ was measured using a spectrometer (Thermo Fisher Scientific, Inc.).

Western blotting. The protein expression levels of ERBB2 were assessed by western blotting. Following treated with different nucleotide fragments as aforementioned, cells were collected and lysed in RIPA buffer (Nanjing KeyGen Biotech Co., Ltd.). Samples were quantified using a Bicinchoninic Acid Assay kit (Beyotime Institute of Biotechnology). Samples (50 $\mu$ g per lane) were separated using $10 \%$ SDS-PAGE and then transferred onto $0.45-\mu \mathrm{m}$ PVDF membranes (Merck KGaA). The membranes were blocked with $5 \%$ skim milk powder solution, followed by incubation overnight at $4^{\circ} \mathrm{C}$ with antibodies against ERBB2 (dilution, 1:1,000; 18299-1-AP; ProteinTech Group, Inc.) and GAPDH (dilution, 1:3,000; 10494-1-AP; ProteinTech Group, Inc.). Subsequently, the membranes were incubated with HRP-conjugated Affinipure Rabbit Anti-Goat IgG(H+L) (dilution, 1:5,000; SA00001-4; ProteinTech Group, Inc.) for $2 \mathrm{~h}$ at $37^{\circ} \mathrm{C}$. Subsequently, bands were detected by incubation with ECL mixture solution (Advansta Inc.), and immediately detected using a Bio-Rad Gel Imaging System (Bio-Rad Laboratories, Inc.). ImageJ software (version 1.8.0; National Institutes of Health) was used to analyze the intensity of the bands.

Reverse transcription-quantitative PCR (RT-qPCR). After undergoing different treatments, cells were collected and lysed using TRIzol (Invitrogen; Thermo Fisher Scientific, Inc.) to extract total RNA. RNA was transcribed into cDNA using a cDNA reverse transcription kit (Takara Bio, Inc.). The conditions used for reverse transcription were as follows: $42^{\circ} \mathrm{C}$ For $15 \mathrm{~min}$, then $5 \mathrm{sec}$ at $85^{\circ} \mathrm{C}$ and storage at $4^{\circ} \mathrm{C}$ for further analysis. Subsequently, cDNA samples were amplified using an RT-qPCR (Applied Biosystems; Thermo Fisher Scientific, Inc.) system and SYBR ${ }^{\circledR}$ Green Premix Ex Taq ${ }^{\mathrm{TM}}$ (Takara Bio, Inc.) according to the manufacturer's protocols. Thermocycling conditions for qPCR were: Pre-denaturation for $30 \mathrm{sec}$ at $95^{\circ} \mathrm{C}$; followed by 40 cycles of $5 \mathrm{sec}$ at $95^{\circ} \mathrm{C}$ and $30 \mathrm{sec}$ at $60^{\circ} \mathrm{C}$; and dissociation at $95^{\circ} \mathrm{C}$ for $15 \mathrm{sec}, 60^{\circ} \mathrm{C}$ for $30 \mathrm{sec}$ and $95^{\circ} \mathrm{C}$ for $15 \mathrm{sec}$. GAPDH and U6 were used as the reference genes to normalize the expression levels of ERBB2 and miR-133a-3p, respectively. mRNA and miRNA expression levels were quantified using the $2^{-\Delta \Delta \mathrm{Cq}}$ method (25). 
The primers used were as follows: ERBB2 forward, 5'-CCA GCCTTCGACAACCTCTATT-3' and reverse, 5'-TGCCGT AGGTGTCCCTTTG-3'; miR-133a-5p forward, 5'-CTTTAA CCATTCTAGCTTTTCCAGGTA-3' and reverse, 5'-GAC TTCGGCTGTGGACAAGATTAG-3'; U6 forward, 5'-CGC TTCGGCAGCACATATACTA-3' and reverse, 5'-CGCTTC ACGAATTTGCGTGTCA-3'; and GAPDH forward, 5'-AGG TCGGTGTGAACGGATTTG-3' and reverse, 5'-GGGGTC GTTGATGGCAACA-3'.

Wound healing assay. The present study measured the cell migration ability using a wound healing assay. After seeding into 24-well plates at the density of $3 \times 10^{5}$ cells per well, H1299 and HCC827 cells were transfected with different nucleotide fragments as aforementioned. After cell reached $80 \%$ confluence, scratches were created across the monolayer using a $10-\mu 1$ pipette tip, followed by washing with cold PBS. An image of each scratch was captured under a light microscope. Subsequently, cells were incubated in RPMI-1640 medium containing 1\% FBS (26). After culturing for $24 \mathrm{~h}$ at $37^{\circ} \mathrm{C}$, images of the scratches were captured again. The migration distance of each group was measured using Image J software, and analyzed with the following formula: (W 0 h-W 24 h)/W 0 h x100\%, where $\mathrm{W}$ is the wound.

Cell invasion assay. A Transwell assay was performed to detect the cell invasion ability following the overexpression of miR-133a-3p or ERBB2. The Transwell inserts were purchased from Corning, Inc. Prior to the experiment, the upper chambers were covered with $100 \mu \mathrm{l}$ of a 1:1 mixture of Matrigel and RPMI-1640 medium and incubated at $37^{\circ} \mathrm{C}$ for $1 \mathrm{~h}$. NSCLC cells were trypsinized, resuspended in serum-free RPMI-1640 medium, and then seeded into the upper chambers $\left(2 \times 10^{4}\right.$ cells/well), while medium containing $20 \%$ FBS was placed in the lower chambers. After culturing at $37^{\circ} \mathrm{C}$ for $24 \mathrm{~h}$, non-invaded cells in the upper chamber were wiped off using sterile cotton swabs. The invaded cells were fixed using $4 \%$ paraformaldehyde at room temperature for $10 \mathrm{~min}$ and stained with $0.1 \%$ crystal violet solution for $20 \mathrm{~min}$ at room temperature. The invaded cells were imaged using an light microscope (magnification, $\mathrm{x} 400$ ).

Dual-luciferase reporter gene assay. Following bioinformatics analysis of the association between miR-133a-3p and the 3'UTR of the ERBB2 transcript (http://www.targetscan. org/vert_72/), the dual-luciferase reporter gene assay was employed using TargetScanHuman 7.2 to verify this prediction. The 3'UTR clones of the ERBB2 transcript, including mutant (MUT) 3'UTRs and wild type (WT) 3'UTRs, were designed and synthesized by Sangon Biotech Co., Ltd. The nucleotide fragments were ligated into the luciferase plasmid (Promega Corporation). H1299 cells were seeded into 24-well plates at the density of $1 \times 10^{5} /$ well, and co-transfected with $300 \mathrm{ng} /$ well plasmid for pmir/ERBB2-WT or pmir/ERBB2-MUT and $50 \mathrm{ng} /$ well miR-133a-3p mimics or miR-negative control (NC) and 100 ng Renilla luciferase plasmid (pRL-TK; Promega Corporation) using Lipofectamine 3000 (Invitrogen; Thermo Fisher Scientific, Inc.), and H1299 cells transfected with pRL-TK alone were regarded as the negative control, followed by incubation for $48 \mathrm{~h}$ at $37^{\circ} \mathrm{C}$. Finally, cells were lysed and the luciferase activities in different groups were detected using a Dual-Luciferase Reporter Assay System (Promega Corporation) following the manufacturer's guidance. Firefly luciferase activities were normalized to Renilla luciferase activities.

Immunofluorescence assay. Immunofluorescence assays were carried out to evaluate E-cadherin and $\mathrm{N}$-cadherin expression. A glass slide was placed in a 6 -well plate, and $2 \times 10^{5}$ cells/well were seeded into the plate. After $24 \mathrm{~h}$, the medium was removed and cells were fixed with $4 \%$ paraformaldehyde at $4^{\circ} \mathrm{C}$ for $30 \mathrm{~min}$. Then, cells were blocked with $10 \%$ goat serum at room temperature $\left(22 \pm 5^{\circ} \mathrm{C}\right)$ for $30 \mathrm{~min}$. Subsequently, cells were incubated with anti-N-cadherin (dilution, 1:1,000; 22018-1-AP; ProteinTech Group, Inc.) or anti-E-cadherin (dilution, 1:1,000; 20874-1-AP; ProteinTech Group, Inc.) antibodies overnight at $4^{\circ} \mathrm{C}$. Afterwards, cells were incubated with the Fluorescein-conjugated Affinipure Goat Anti-Rabbit IgG (H+L) secondary antibody (dilution, 1:100; SA00003-2; ProteinTech Group, Inc.) at room temperature for $1 \mathrm{~h}$, followed by staining using DAPI at room temperature for $5 \mathrm{~min}$. Finally, images were captured using a fluorescence microscope (magnification, $\mathrm{x} 400$ ).

Statistical analysis. The data analysis in the present study was conducted using SPSS 23.0 (IBM Corp.) and GraphPad 6.0 software (GraphPad Software, Inc.). Student's t-test was carried out to compare differences between two groups. Statistical differences among three groups were analyzed using one-way ANOVA followed by Duncan's post hoc test (LSR) at the $95 \%$ confidence interval $(\mathrm{P}<0.05$ was considered to indicate statistical significance). Statistical differences among four groups were analyzed using one-way ANOVA followed by Tukey's post hoc test. $\mathrm{P}<0.05$ was considered to indicate a statistically significant difference. Data were expressed as mean \pm standard deviation. Each assay was repeated three times.

\section{Results}

Expression levels of miR-133a-3p and ERBB2 are dysregulated in NSCLC tissues and cell lines. The present study assessed miR133a-3p expression at the mRNA level and ERBB2 expression at the mRNA and protein levels in NSCLC tissues and normal adjacent tissues. As shown in Fig. 1A, RT-qPCR results revealed that miR-133a-3p expression was markedly downregulated in NSCLC tissues compared with adjacent healthy tissues, while the mRNA expression levels of ERBB2 were upregulated in cancer tissues. Western blotting demonstrated that the protein expression levels of ERBB2 were elevated in NSCLC tissues (Fig. 1B).

In addition, the present study evaluated the expression levels of these molecules in NSCLC cells. Consistent with the results identified in tissue samples, lower miR-133a-3p expression was observed in NSCLC cells (H1299 and HCC827 cell lines), while ERBB2 expression was upregulated both at the mRNA and protein levels compared with that in the normal pulmonary epithelial cells (BEAS-2B; Fig. 1C and D). These results indicated that miR-133a-3p expression was decreased, while ERBB2 expression was increased in NSCLC tissues and cells. 


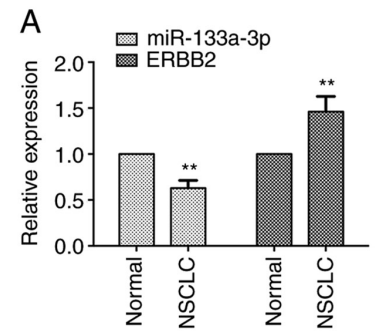

B
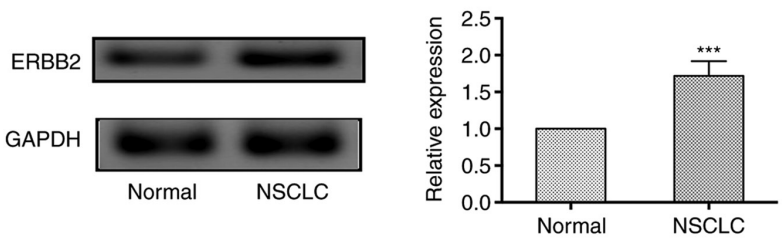

C

$\mathrm{D}$
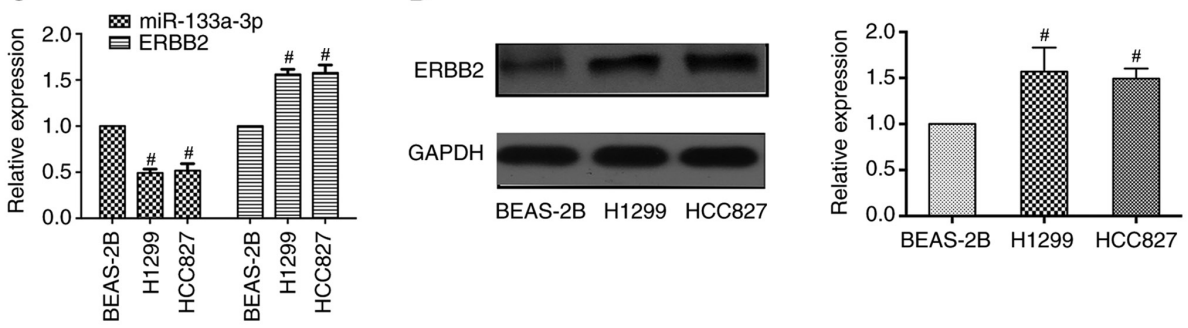

Figure 1. miR-133a-3p and ERBB2 expression are dysregulated in both NSCLC tissues and cell lines. (A) miR-133a-3p expression was downregulated in cancer tissues at the mRNA level, whereas ERBB2 expression was upregulated in cancer tissues compared with in normal tissues. (B) ERBB2 protein expression was increased in NSCLC tissues. (C) miR-133a-3p expression was downregulated in NSCLC cell lines (H1299 and HCC827) at the mRNA level, while ERBB2 expression was upregulated. (D) ERBB2 expression was increased in NSCLC cell lines at the protein level. ${ }^{* *} \mathrm{P}<0.01$ and ${ }^{* * *} \mathrm{P}<0.001 \mathrm{vs}$. normal. ${ }^{*} \mathrm{P}<0.05$ vs. BEAS-2B. ERBB2, erb-b2 receptor tyrosine kinase 2; miR-133a-3p, microRNA-133a-3p; NSCLC, non-small cell lung cancer.

Overexpression of miR-133a-3p inhibits NSCLC cell proliferation, migration and invasion. To determine the biological role of miR-133a-3p, H1299 and HCC827 cells were transfected with control or miR-133a-3p mimic. RT-qPCR was performed to evaluate transfection efficiency, which demonstrated that miR-133a-3p expression was significantly increased after transfection with miR-133a-3p mimic (Fig. 2A). An MTT assay was used to assess the effect of miR-133a-3p on NSCLC cell proliferation. As shown in Fig. 2B, overexpression of miR-133a-3p led to a suppression of proliferation in NSCLC cells. The Transwell assay indicated that overexpression of miR133a-3p inhibited the invasion abilities of these cells (Fig. 2C). In addition, the wound healing assay indicated the inhibitory effects of miR-133a-3p on migration (Fig. 2D). Therefore, the present results demonstrated that overexpression of miR-133a-3p inhibited the proliferation, migration and invasion of NSCLC cells. Furthermore, RT-qPCR and western blotting data indicated that the mRNA and protein expression levels of ERBB2 were decreased in the miR-133a-3p mimics group, compared with mimics control groups (Fig. 3).

Overall, upregulation of ERBB2 and decreased expression levels of miR-133a-3p were observed in NSCLC tissues and cell lines. Downregulation of ERBB2 expression was observed in cells transfected with miR-133a-3p mimic, indicating that ERBB2 may be an indirect or direct target of miR-133a-3p.

miR-133a-3p directly targets the 3'UTR of the ERBB2 transcript in NSCLC cells. As aforementioned, a negative association between ERBB2 and miR-133-3p expression was noted. To further analyze this interaction, bioinformatics analysis was performed using TargetScan (Fig. 4A). This suggested that the 3 'UTR of the ERBB2 transcript is a potential target of miR-133a-3p. A dual-luciferase reporter assay was performed to verify this prediction. As shown in Fig. 4B, miR-133a-3p overexpression markedly suppressed the luciferase activity in cells transfected with pmir/ERBB2-WT, while the luciferase activity was not changed in cells transfected with pmir/ERBB2-MUT. Therefore, these data provide evidence that miR-133a-3p directly targets the 3'UTR of the ERBB2 transcript in NSCLC cells.

miR-133a-3p inhibits the proliferation, invasion and migration of NSCLC cells by negatively regulating ERBB2 expression. To further explore the biological functions of the miR-133a-3p/ERBB2 axis in NSCLC progression, H1299 cells were treated with miR-133a-3p mimic, pcDNA-ERBB2 or miR-133a-3p mimic + pcDNA-ERBB2. pcDNA-NC and pcDNA-ERBB2 were transfected into H1299 cells, and pcDNA-NC did not affect the expression levels of ERBB2 in H1299 cells (Fig. S1).

RT-qPCR and western blotting were performed to detect miR-133a-3p and ERBB2 expression in each treatment group. As shown in Fig. 5A, miR-133a-3p expression was increased in both the miR-133a-3p mimic and miR-133a-3p mimic + pcDNA-ERBB2 groups but was not significantly altered in the pcDNA-ERBB2 group compared with the $\mathrm{NC}$ group. Furthermore, ERBB2 expression was increased in the pcDNA-ERBB2 group, while it was decreased in the miR-133a-3p mimics group, compared with non-treated (NC) group. Compared with the pcDNA-ERBB2 group, ERBB2 expression was suppressed in the miR-133a-3p mimic + pcDNA-ERBB2 group (Fig. 5B).

MTT, Transwell and wound healing assays were performed to demonstrate the potential role of the miR-133a-3p/ERBB2 axis in NSCLC cell phenotypes. As shown in Fig. 5C, the MTT data demonstrated that overexpression of miR-133a-3p and ERBB2 in combination preserved the proliferation activity of H1299 cells, which was suppressed in miR-133a-3p mimics group. Compared with pcDNA-ERBB2 group, the proliferation of H1299 cells in miR-133a-3p 

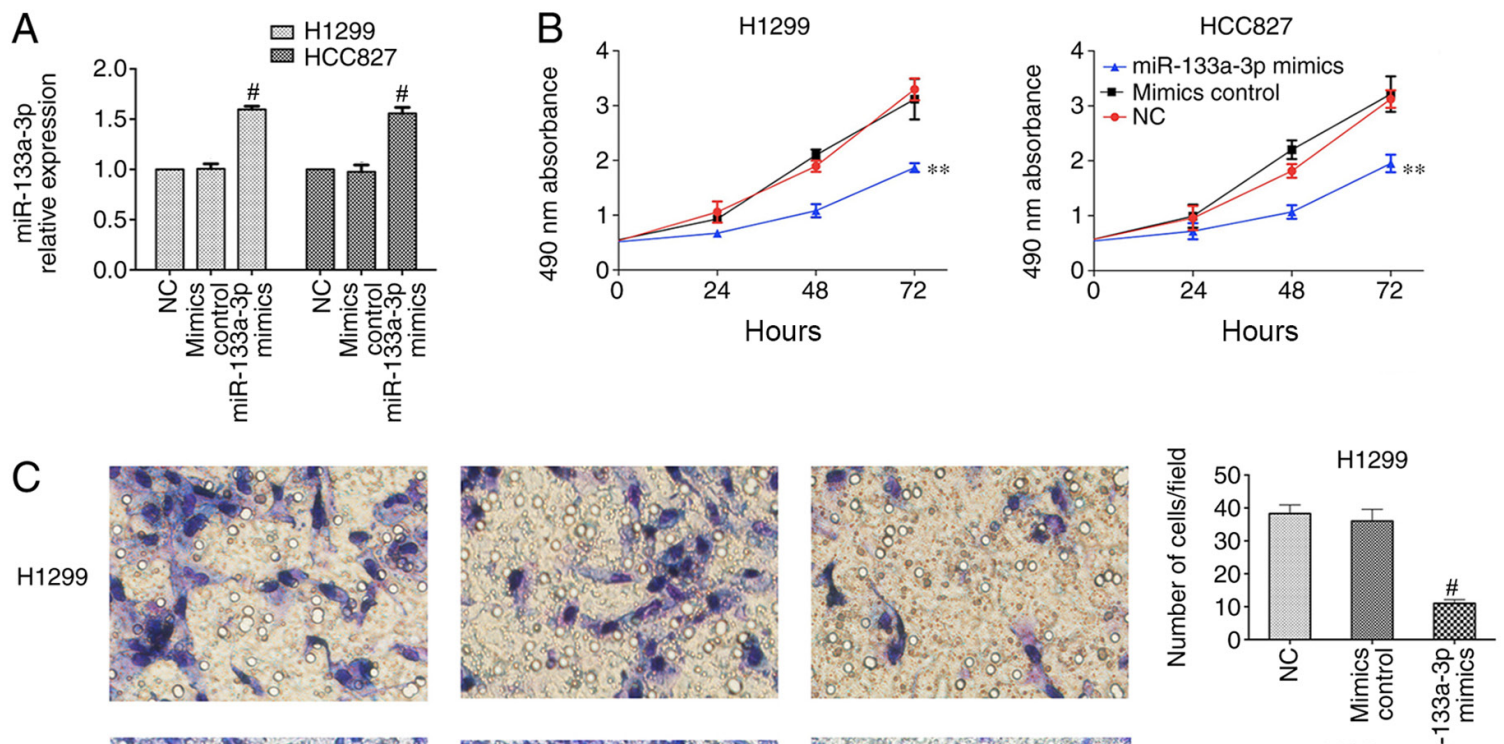

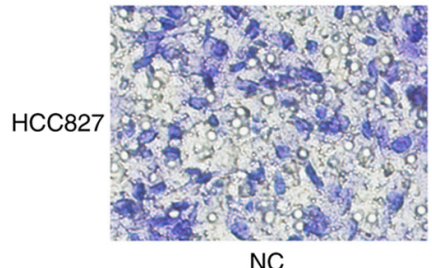

NC

D
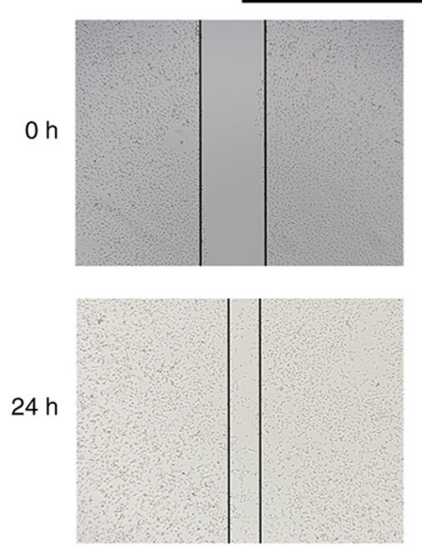

NC

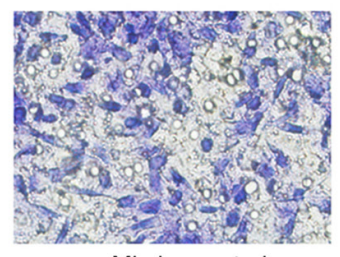

Mimics control

$\mathrm{H} 1299$
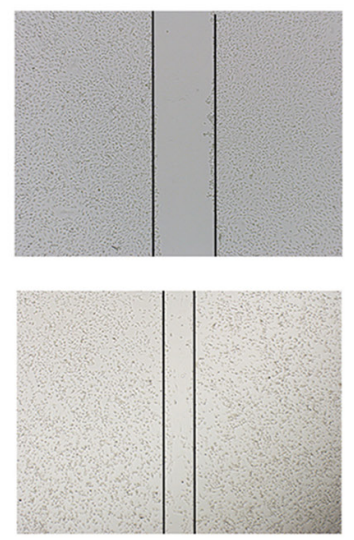

Mimics control

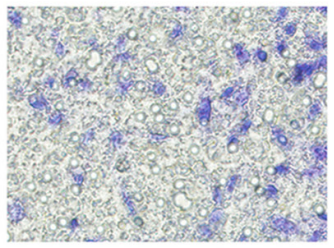

miR-133a-3p mimics
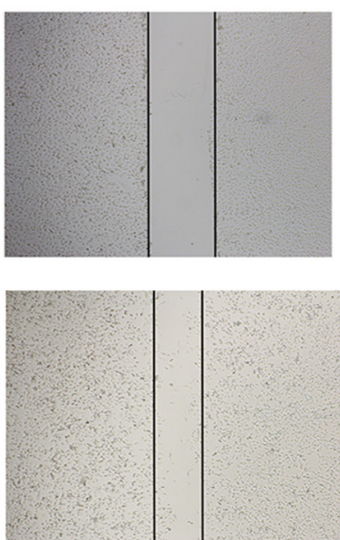

miR-133a-3p mimics
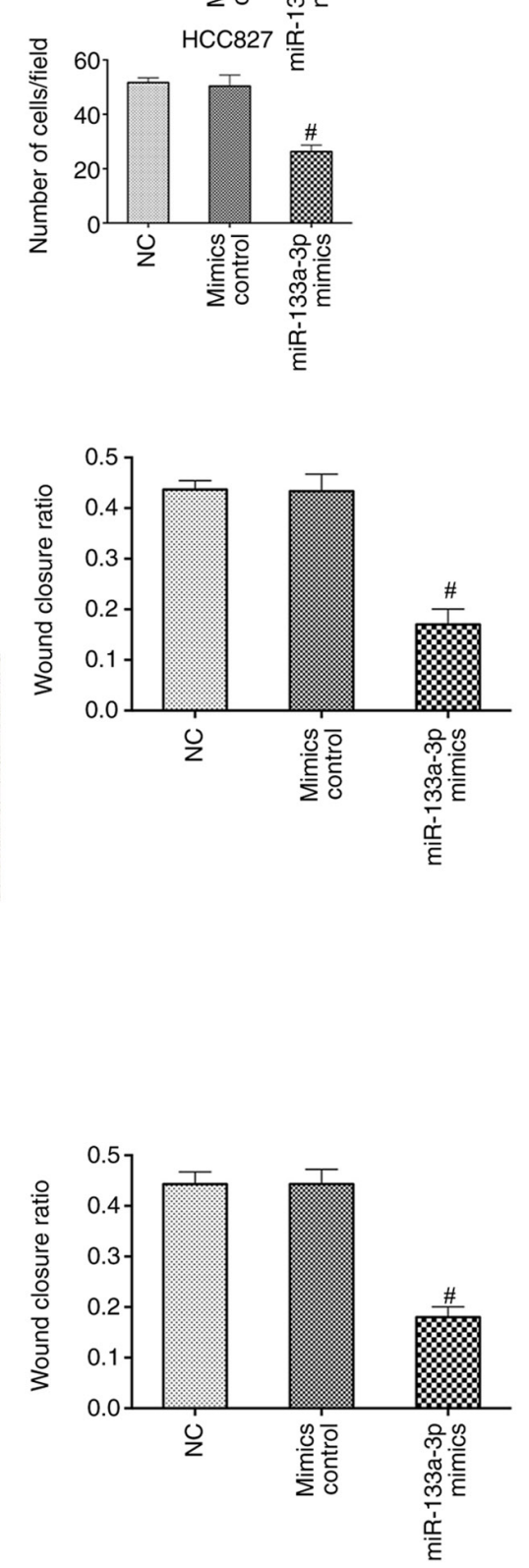

NC
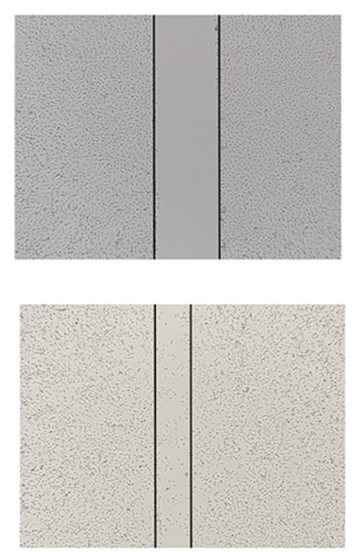

Mimics control
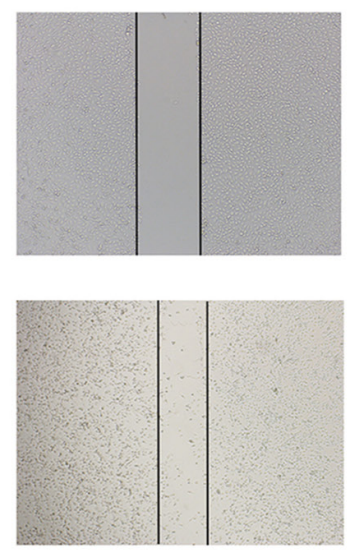

miR-133a-3p mimics

Figure 2. Overexpression of miR-133a-3p inhibits non-small cell lung cancer cell proliferation, migration and invasion. (A) miR-133a-3p mimics transfection successfully increased the miR-133a-3p levels in H1299 and HCC827 cells. (B) Proliferation was suppressed in cells transfected with miR-133a-3p mimics. (C) Invasion abilities were suppressed in cells transfected with miR-133a-3p mimics. Magnification, $x 400$. (D) Migration abilities were inhibited in cells following overexpression of miR-133a-3p. Magnification, $x 400 .{ }^{* *} \mathrm{P}<0.01$ vs. NC. ${ }^{\#} \mathrm{P}<0.05$ vs. NC. miR-133a-3p, microRNA-133a-3p; NC, negative control. 
A

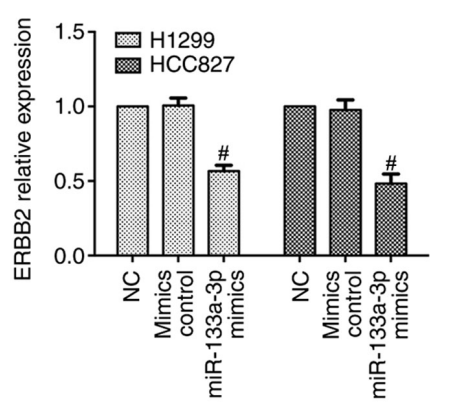

B

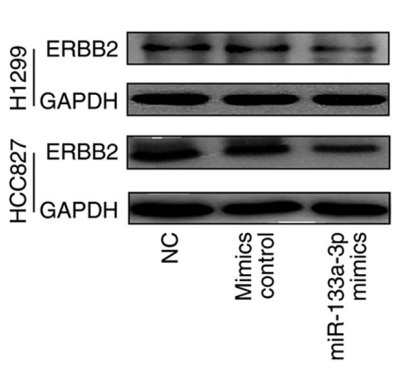

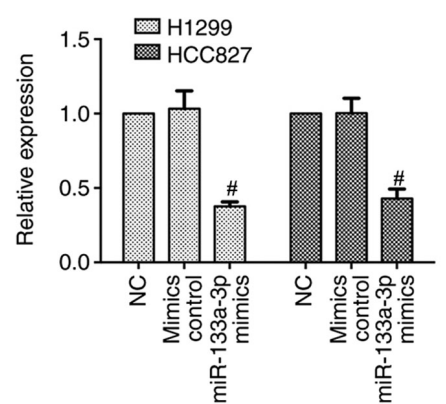

Figure 3. Overexpression of miR-133a-3p suppresses ERBB2 expression. (A) ERBB2 expression was decreased in non-small cell lung cancer cell lines (H1299 and $\mathrm{HCC} 827$ ) at the mRNA level following miR-133a-3p mimics treatment. (B) ERBB2 protein expression was decreased after miR-133a-3p mimic transfection. ${ }^{~} \mathrm{P}<0.05$ vs. NC. ERBB2, erb-b2 receptor tyrosine kinase 2; miR-133a-3p, microRNA-133a-3p; NC, negative control.

A

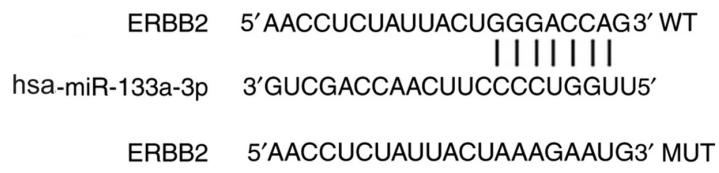

B

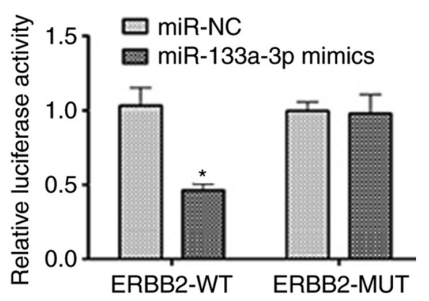

Figure 4. miR-133a-3p directly targets the 3' untranslated region of the ERBB2 transcript in non-small cell lung cancer cells. (A) Bioinformatics analysis of miR-133a-3p and ERBB2. (B) Co-transfection of miR-133a-3p and pmir/ERBB2-WT markedly suppressed the luciferase activity. "P<0.05 vs. miR-NC. ERBB2, erb-b2 receptor tyrosine kinase 2; miR-133a-3p, microRNA-133a-3p; NC, negative control; WT, wild type; MUT, mutant.

mimics+pcDNA-ERBB2 group decreased. In addition, the invasive ability was attenuated in H1299 cells transfected with both miR-133a-3p mimics and pcDNA-ERBB2 (miR-133a-3p mimics+pcDNA-ERBB2 group), compared with pcDNA-ERBB2 group (Fig. 5D). Finally, the wound healing assay suggested that the co-transfection of miR-133a-3p mimic and pcDNA-ERBB2 decreased the wound closure ratio in H1299 cells compared with that in cells in the pcDNA-ERBB2 group (Fig. 5E). These results suggest that miR-133a-3p inhibits cell proliferation, invasion and migration of NSCLC cells by negatively regulating ERBB2 expression.

Overexpression of miR-133a-3p regulates the expression levels of epithelial-mesenchymal transition (EMT) biomarkers. $\mathrm{E}$-cadherin and $\mathrm{N}$-cadherin are two important biomarkers of the EMT process. The present study detected E-cadherin and $\mathrm{N}$-cadherin expression using an immunofluorescence assay following transfection with miR-133a-3p mimics, pcDNA-ERBB2 or miR-133a-3p mimics + pcDNA-ERBB2. As shown in Fig. 6A, N-cadherin expression was decreased in the miR-133a-3p mimics group but increased in the pcDNA-ERBB2 group. Consistently, E-cadherin exhibited the opposite trends (Fig. 6B). These results indicate that the overexpression of miR-133a-3p suppresses the EMT process in NSCLC cells.

\section{Discussion}

At present, surgery and chemotherapy are the mainstream treatment modalities for NSCLC (27). However, the outcome of these treatments is unsatisfactory, since postoperative recurrence and therapy resistance are common in patients with NSCLC (28). With the development of molecular biology, targeted therapy has become available for malignant tumors (29). However, its efficacy remains limited, since most patients eventually develop drug resistance. Therefore, there is still an urgent need for the identification of novel therapeutic targets. In the last decade, miRNAs have become a hot topic in cancer diagnosis and treatment (18). miRNAs are a type of short, non-coding RNA molecules that serve critical regulatory roles in multiple cellular events. Increasing evidence has suggested that miRNA dysregulation is closely associated with the pathogenesis and progression of a variety of diseases, in particular cancer types (30), for example miR-133a-3p expression is downregulated in gastric cancer (21). With the increased understanding of miRNAs, the feasibility of using miRNAs as therapeutic targets is supported $(31,32)$.

Previous studies have suggested a tumor-suppressive role of miR-133a-3p in multiple malignant tumors, including gastric, breast and bladder cancer $(21,22,33)$. Low expression levels of miR-133a-3p are associated with poor prognosis and faster progression (22). Shi et al (33) reported that downregulation of miR-133a-3p promotes the proliferation and invasion of breast cancer cells. Li et al (34) suggested that miR-133a-3p promotes autophagy in gastric cancer cells by negatively regulating forehead box P3. The present study identified decreased miR-133a-3p expression in NSCLC cancer tissues and cell lines. A series of cellular and molecular experiments were performed to identify its biological function in NSCLC pathogenesis. The present results indicated that overexpression 

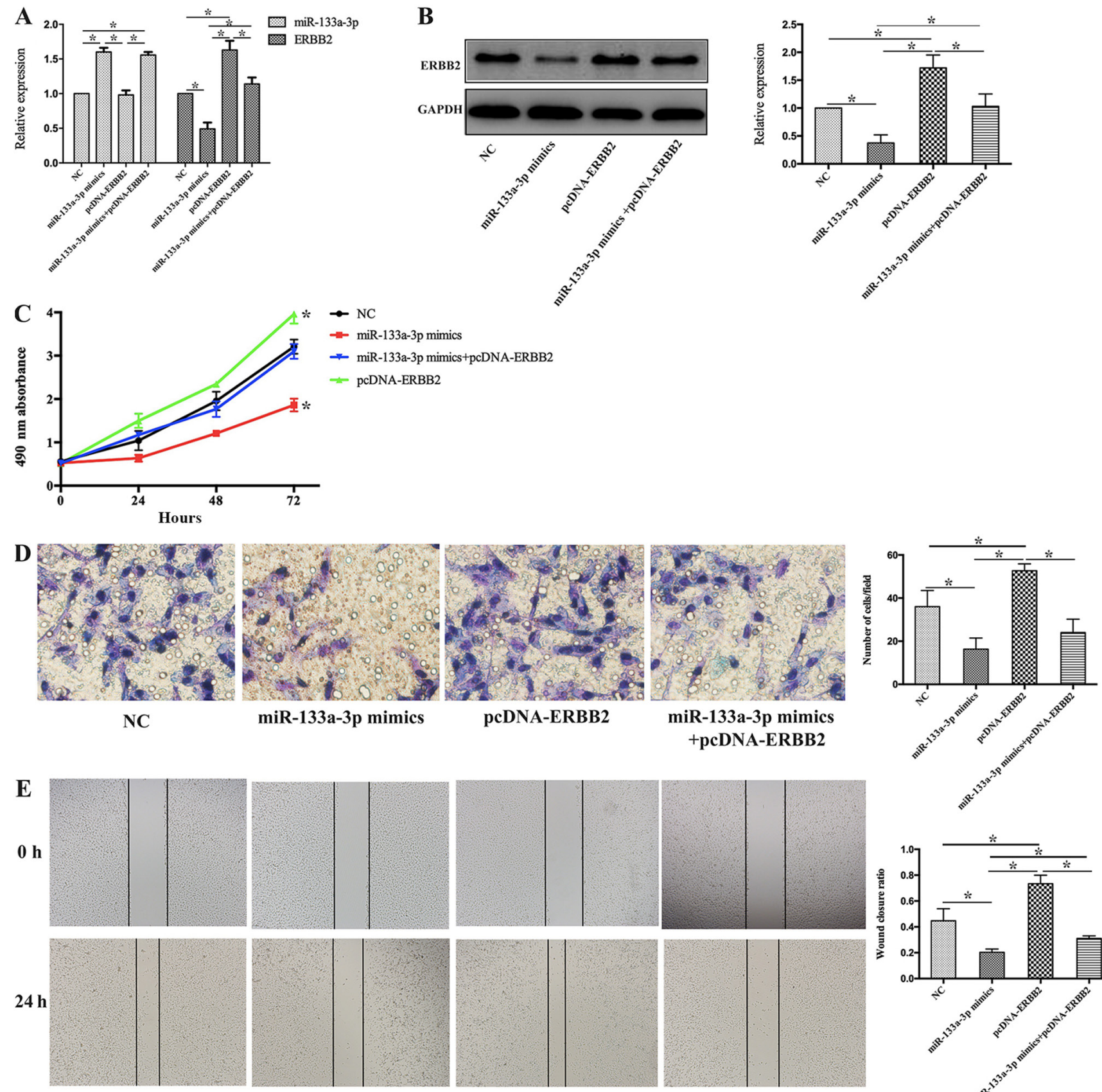

pcDNA-ERBB2
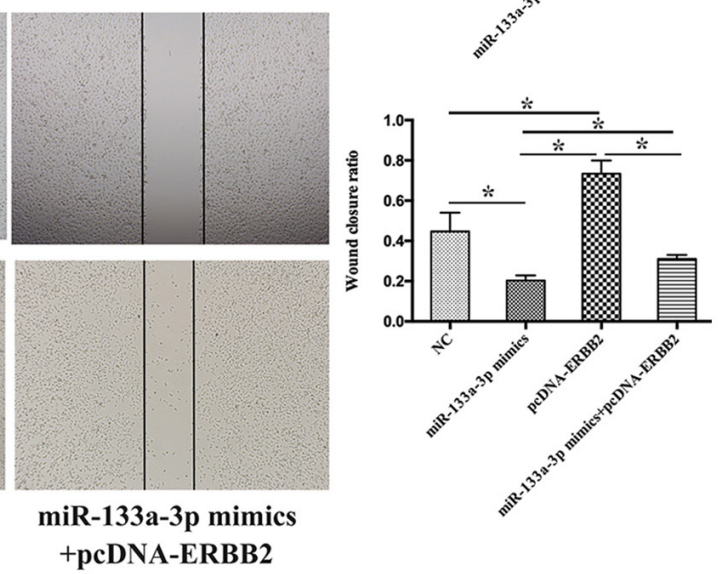

Figure 5. miR-133a-3p inhibits the proliferation, invasion and migration of non-small cell lung cancer cells by negatively regulating ERBB2. (A) At the mRNA level, the expression levels of miR-133a-3p were increased in the miR-133a-3p mimic and miR-133a-3p mimic + pcDNA-ERBB2 groups. ERBB2 expression was increased in the pcDNA-ERBB2 group, while it was decreased in cells transfected with miR-133a-3p mimic. (B) ERBB2 protein expression was suppressed in cells transected with miR-133a-3p mimic. (C) MTT assay data demonstrated that upregulated miR-133a-3p and ERBB2 in combination preserved the H1299 cell proliferation activity compared with cells transfected with pcDNA-ERBB2 alone. (D) The invasion ability was attenuated in H1299 cells transfected with miR-133a-3p mimic and pcDNA-ERBB2 in combination compared with cells transfected with pcDNA-ERBB2 alone. Magnification, x400. (E) Wound healing assays suggested that co-transfection with miR-133a-3p mimic and pcDNA-ERBB2 decreased the wound closure ratio in H1299 cells compared with transfection with pcDNA-ERBB2 alone. Magnification, $x 400 .{ }^{*} \mathrm{P}<0.05$ vs. NC. ERBB2, erb-b2 receptor tyrosine kinase 2; miR-133a-3p, microRNA-133a-3p; $\mathrm{NC}$, negative control.

of miR-133a-3p effectively suppressed the cell proliferation and invasion of NSCLC cells.

To further assess the molecular mechanisms underlying the tumor-suppressive effect of miR-133a-3p, bioinformatics analysis was performed using TargetScan 7.2. Among the potential target genes, the present study focused on ERBB2, a well-known oncogene in NSCLC (35). ERBB2 is a member of the EGFR family, which is involved in a series of biological events in malignant diseases (36). It has been suggested that ERBB2 could trigger several cellular processes, including cell proliferation, survival and differentiation $(8,37)$. Spencer et al (38) provided evidence that the ERBB2 receptor assists the binding of p130Cas to CRK, and thus, promotes the invasion and migration of breast cancer cells. Furthermore, 
A

miR-133a-3p mimics

pCDNA-ERBB2

miR-133a-3p mimics+

NC
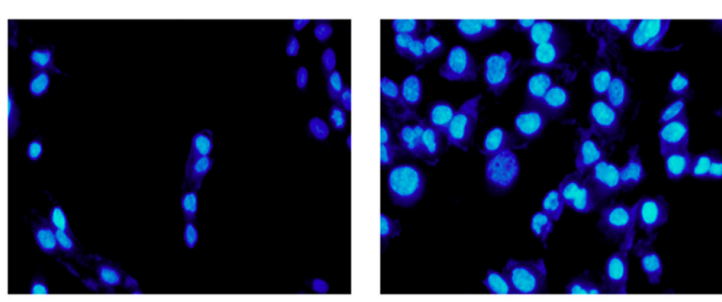

pcDNA-ERBB2
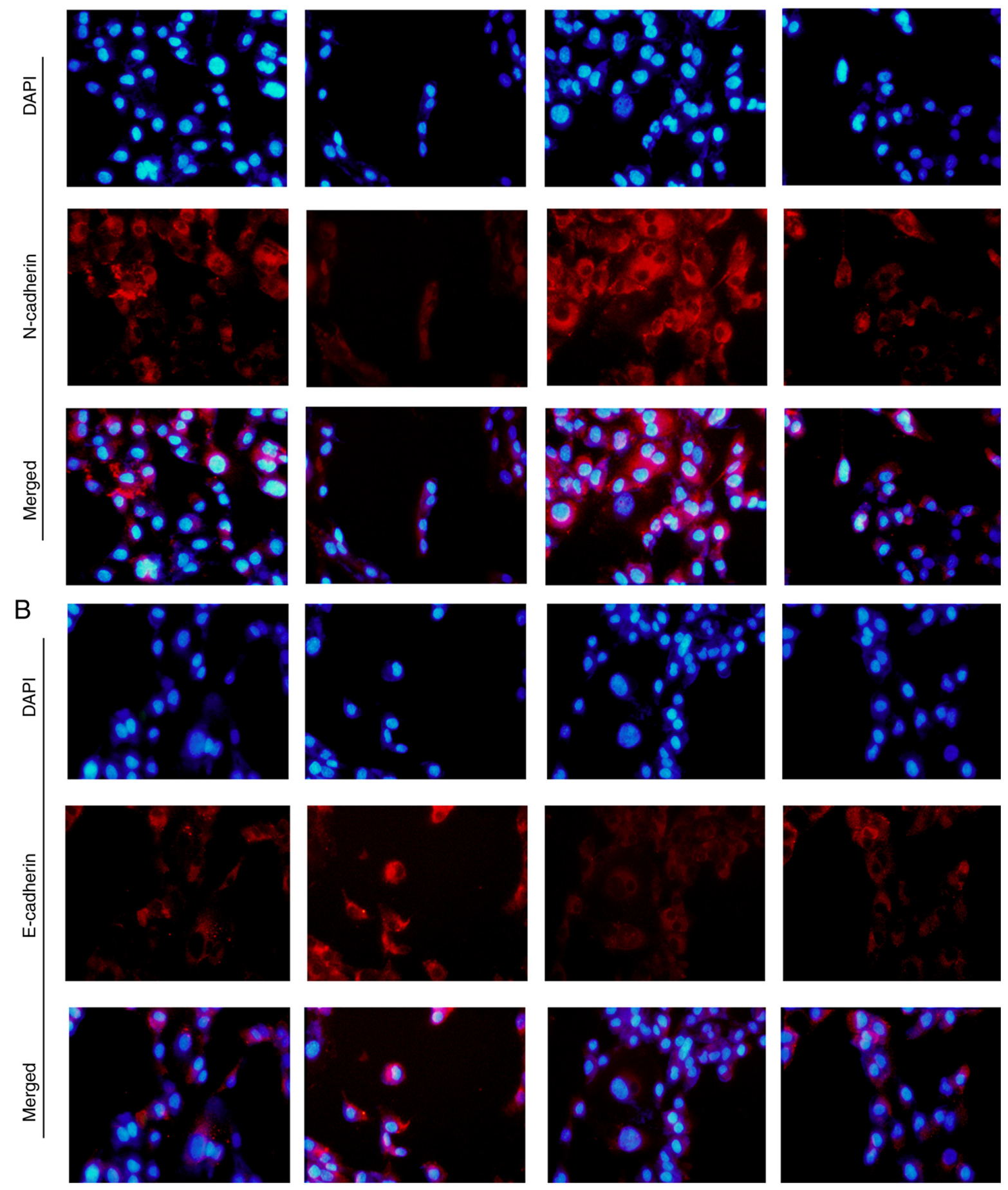

Figure 6. Overexpression of miR-133a-3p regulates the expression levels of epithelial-mesenchymal transition biomarkers. (A) N-cadherin expression was decreased in the miR-133a-3p group, while it was increased in the pcDNA-ERBB2 group. Overexpression of miR-133a-3p and ERBB2 rescued the expression levels of N-cadherin. (B) E-cadherin expression was increased in the miR-133a-3p group, whereas it was decreased in the pcDNA-ERBB2 group. Overexpression of miR-133a-3p and ERBB2 rescued the expression levels of E-cadherin. Magnification, x400. ERBB2, erb-b2 receptor tyrosine kinase 2; miR-133a-3p, microRNA-133a-3p; NC, negative control.

ERBB2 expression is upregulated in various malignancies, and ERBB2-targeted therapy has become an important component of therapeutic strategies in a number of cancer types (12). In the present study, the luciferase assay supported the notion that miR-133a-3p could target the 3'UTR of ERBB2. Furthermore, a pcDNA-ERBB2 vector was employed to increase ERBB2 expression, and this reversed the effects of the miR-133a-3p mimic on cell proliferation, invasion and migration. Therefore, the tumor-suppressive effect of miR-133a-3p may be mediated, at least in part, by inhibition of ERBB2 expression.

EMT is an essential event in tumor carcinogenesis and progression. As a member of the EGFR family, ERBB2 has 
been demonstrated to promote EMT in previous study (39). The present study detected the typical EMT biomarkers E-cadherin and $\mathrm{N}$-cadherin and demonstrated that overexpression of miR-133a-3p markedly suppressed the EMT process in NSCLC cells, while overexpression of ERBB2 ameliorated this effect. Therefore, the present findings suggest that miR-133a-3p may suppress EMT by targeting ERBB2.

There were some limitations to the present study. Commonly in wound healing assays non-FBS medium should be used; however, $1 \%$ FBS was used in the present study to prevent cell starving. Also, the present study only provided in vitro data, therefore further in vivo experiments, such as tumorigenesis assay, should be performed in future. Besides, further analysis of whether miR-133a-3p can regulate other signaling pathways impacting the phenotypes of NSCLC are needed.

In conclusion, the present results indicate that miR-133a-3p acts as a tumor-suppressive gene via inhibition of the proliferation, invasion and EMT of NSCLC cells. In particular, the inhibitory effects of miR-133a-3p on ERBB2 indicated that miR-133a-3p may be a potent antitumor target for NSCLC treatment.

\section{Acknowledgements}

Not applicable.

\section{Funding}

No funding was received.

\section{Availability of data and materials}

The datasets used and/or analyzed during the present study are available from the corresponding author on reasonable request.

\section{Authors' contributions}

The majority of experiments and manuscript were finished by YX. YX performed statistical analysis and interpreted the data. LZ contribute to bioinformatics analysis and dual luciferase reporter gene experiment. LX and XZ designed the research, collected the tissues and edited the manuscript. All authors read and approved the final manuscript.

\section{Ethics approval and consent to participate}

The present study was conducted in accordance with the Helsinki Declaration guidelines, and written consent to participation in the present study were obtained from all patients or their direct relatives. Furthermore, the present study was approved by The Ethics Committee of Zhejiang Hospital (Hangzhou, China; approval no. 20100103).

\section{Patient consent for publication}

Not applicable.

\section{Competing interests}

The authors declare that they have no competing interests.

\section{References}

1. Duffy SW and Field JK: Mortality reduction with low-dose CT screening for lung cancer. N Engl J Med 382: 572-573, 2020.

2. Qi M, Dai D, Liu J, Li Z, Liang P, Wang Y, Cheng L, Zhan Y, An Z, Song Y, et al: AIM2 promotes the development of non-small cell lung cancer by modulating mitochondrial dynamics. Oncogene 39: 2707-2723, 2020.

3. Siegel RL, Miller KD and Jemal A: Cancer statistics, 2015. CA Cancer J Clin 65: 5-29, 2015.

4. Zhang H, Qian G, Zong D, Fan S, Owonikoko TK, Ramalingam SS and Sun SY: Overcoming acquired resistance of epidermal growth factor receptor-mutant non-small cell lung cancer cells to osimertinib by combining osimertinib with the histone deacetylase inhibitor panobinostat (LBH589). Cancer 126: 2024-2033, 2020.

5. Morgensztern D, Ng SH, Gao F and Govindan R: Trends in stage distribution for patients with non-small cell lung cancer: A national cancer database survey. J Thorac Oncol 5: 29-33, 2010.

6. Du L and Morgensztern D: Chemotherapy for advanced-stage non-small cell lung cancer. Cancer J 21: 366-370, 2015.

7. Zhang Q, Zhou L, Guan Y, Cheng Y and Han X: BENC-511, a novel PI3K inhibitor, suppresses metastasis of non-small cell lung cancer cells by modulating $\beta$-catenin/ZEB1 regulatory loop. Chem Biol Interact 294: 18-27, 2018.

8. Yang ZY, Yang L, Xu CW, Wang XJ and Lei L: An insertion mutation of ERBB2 enhances breast cancer cell growth and confers resistance to lapatinib through AKT signaling pathway. Biol Open 9: bio047662, 2020.

9. Honkoop H, de Bakker DE, Aharonov A, Kruse F, Shakked A, Nguyen PD, de Heus C, Garric L, Muraro MJ, Shoffner A, et al: Single-cell analysis uncovers that metabolic reprogramming by ErbB2 signaling is essential for cardiomyocyte proliferation in the regenerating heart. Elife 8: e50163, 2019.

10. Albrecht T, Rausch M, Rössler S, Albrecht M, Braun JD, Geissler V, Mehrabi A, Vogel MN, Pathil-Warth A, Mechtersheimer G, et al: HER2 gene (ERBB2) amplification is a rare event in non-liver-fluke associated cholangiocarcinogenesis. BMC Cancer 19: 1191, 2019.

11. Menderes G, Bonazzoli E, Bellone S, Black J, Altwerger G, Masserdotti A, Pettinella F, Zammataro L, Buza N, Hui P, et al: SYD985, a novel duocarmycin-based HER2-targeting antibody-drug conjugate, shows promising antitumor activity in epithelial ovarian carcinoma with HER2/Neu expression. Gynecol Oncol 146: 179-186, 2017.

12. Ahmed S, Sami A and Xiang J: HER2-directed therapy: Current treatment options for HER2-positive breast cancer. Breast Cancer 22: 101-116, 2015.

13. Li L, Jia L and Ding Y: Upregulation of miR-375 inhibits human liver cancer cell growth by modulating cell proliferation and apoptosis via targeting ErbB2. Oncol Lett 16: 3319-3326, 2018.

14. Rimawi MF, Schiff R and Osborne CK: Targeting HER2 for the treatment of breast cancer. Annu Rev Med 66: 111-128, 2015.

15. Xiang Z, Huang X, Wang J, Zhang J, Ji J, Yan R, Zhu Z, Cai W and $\mathrm{Yu} Y$ : Cross-database analysis reveals sensitive biomarkers for combined therapy for ERBB2 ${ }^{+}$gastric cancer. Front Pharmacol 9: 861, 2018.

16. Wu J, Li S, Ma R, Sharma A, Bai S, Dun B, Cao H, Jing C, She J and Feng J: Tumor profiling of co-regulated receptor tyrosine kinase and chemoresistant genes reveal different targeting options for lung and gastroesophageal cancers. Am J Transl Res 8: 5729-5740, 2016.

17. Liang CH, Shiu LY, Chang LC, Sheu HM and Kuo KW: Solamargine upregulation of Fas, downregulation of HER2, and enhancement of cytotoxicity using epirubicin in NSCLC cells. Mol Nutr Food Res 51: 999-1005, 2007.

18. Lu TX and Rothenberg ME: MicroRNA. J Allergy Clin Immunol 141: 1202-1207, 2018.

19. Olejniczak M, Kotowska-Zimmer A and Krzyzosiak W: Stress-induced changes in miRNA biogenesis and functioning. Cell Mol Life Sci 75: 177-191, 2018.

20. Ohanian M, Humphreys DT, Anderson E, Preiss T and Fatkin D: A heterozygous variant in the human cardiac miR-133 gene, MIR133A2, alters miRNA duplex processing and strand abundance. BMC Genet 14: 18, 2013.

21. Zhang X, Li Z, Xuan Z, Xu P, Wang W, Chen Z, Wang S, Sun G, $\mathrm{Xu} \mathrm{J}$ and $\mathrm{Xu} \mathrm{Z}$ : Novel role of miR-133a-3p in repressing gastric cancer growth and metastasis via blocking autophagy-mediated glutaminolysis. J Exp Clin Cancer Res 37: 320, 2018. 
22. Gao L, Li SH, Tian YX, Zhu QQ, Chen G, Pang YY and Hu XH: Role of downregulated miR-133a-3p expression in bladder cancer: A bioinformatics study. Onco Targets Ther 10: 3667-3683, 2017.

23. He B, Lin X, Tian F, Yu W and Qiao B: miR-133a-3p inhibits ora squamous cell carcinoma (OSCC) proliferation and invasion by suppressing COL1A1. J Cell Biochem 119: 338-346, 2018.

24. Yang ZQ, Wu CA and Cheng YX: Prognostic value of microRNA-133a expression and its clinicopathologic significance in non-small cell lung cancer: A comprehensive study based on meta-analysis and the TCGA database. Oncol Res Treat 41: 762-768, 2018.

25. Livak KJ and Schmittgen TD: Analysis of relative gene expression data using real-time quantitative PCR and the 2(-Delta Delta C(T)) method. Methods 25: 402-408, 2001.

26. Tian L, Chen M, He Q, Yan Q and Zhai C: MicroRNA-199a-5p suppresses cell proliferation, migration and invasion by targeting ITGA3 in colorectal cancer. Mol Med Rep 22: 2307-2317, 2020.

27. Gamerith G, Kocher F, Rudzki J and Pircher A: ASCO 2018 NSCLC highlights-combination therapy is key. Memo 11: 266-271, 2018.

28. Tian Y, Sun C, Zhang L and Pan Y: Clinical significance of miRNA-106a in non-small cell lung cancer patients who received cisplatin combined with gemcitabine chemotherapy. Cancer Biol Med 15: 157-164, 2018.

29. $\mathrm{Hu}$ Y, Liu C and Muyldermans S: Nanobody-based delivery systems for diagnosis and targeted tumor therapy. Front Immunol 8: 1442, 2017.

30. McGuire A, Brown JA and Kerin MJ: Metastatic breast cancer: The potential of miRNA for diagnosis and treatment monitoring. Cancer Metastasis Rev 34: 145-155, 2015.

31. Shin VY and Chu KM: miRNA as potential biomarkers and therapeutic targets for gastric cancer. World J Gastroenterol 20 : 10432-10439, 2014.
32. Takahashi RU, Prieto-Vila M, Kohama I and Ochiya T: Development of miRNA-based therapeutic approaches for cancer patients. Cancer Sci 110: 1140-1147, 2019.

33. Shi W, Tang T, Li X, Deng S, Li R, Wang Y, Wang Y, Xia T, Zhang Y, Zen K, et al: Methylation-mediated silencing of miR-133a-3p promotes breast cancer cell migration and stemness via miR-133a-3p/MAML1/DNMT3A positive feedback loop. J Exp Clin Cancer Res 38: 429, 2019.

34. Li JP, Zhang HM, Liu MJ, Xiang Y, Li H, Huang F, Li HH, Dai ZT, Gu CJ, Liao XH and Zhang TC: miR-133a-3p/FOXP3 axis regulates cell proliferation and autophagy in gastric cancer. J Cell Biochem 121: 3392-3405, 2020.

35. Byeon S, Lee B, Park WY, Choi YL, Jung HA, Sun JM, Ahn JS, Ahn MJ, Park K and Lee SH: Benefit of targeted DNA sequencing in advanced non-small-cell lung cancer patients without EGFR and ALK alterations on conventional tests. Clin Lung Cancer 21: e182-e190, 2020.

36. Liberelle M, Jonckheere N, Melnyk P, Van Seuningen I and Lebègue N: EGF-containing membrane-bound mucins: A hidden ErbB2 targeting pathway? J Med Chem 63: 5074-5088, 2020.

37. Voelker R: Another targeted therapy for ERBB2-positive breast cancer. JAMA 323: 408, 2020.

38. Spencer KS, Graus-Porta D, Leng J, Hynes NE and Klemke RL: ErbB2 is necessary for induction of carcinoma cell invasion by ErbB family receptor tyrosine kinases. J Cell Biol 148: 385-397, 2000.

39. Jung DH, Bae YJ, Kim JH, Shin YK and Jeung HC: HER2 regulates cancer stem cell activities via the Wnt signaling pathway in gastric cancer cells. Oncology 97: 311-318, 2019. 